\title{
Article \\ Comparing Sampling Strategies for Tackling Imbalanced Data in Human Activity Recognition
}

\author{
Fayez Alharbi ${ }^{1,2, *}$, Lahcen Ouarbya ${ }^{2}$ and Jamie A Ward ${ }^{2}$ (1) \\ 1 Computer Sciences and Information Technology College, Majmaah University, \\ Al Majmaah 15341, Saudi Arabia \\ 2 Department of Computing, Goldsmiths, University of London, London SE14 6NW, UK; \\ l.ouarbya@gold.ac.uk (L.O.); j.ward@gold.ac.uk (J.A.W.) \\ * Correspondence: fs.alharbi@mu.edu.sa
}

Citation: Alharbi, F.; Ouarbya, L.; Ward, J.A. Comparing Sampling Strategies for Tackling Imbalanced Data in Human Activity Recognition. Sensors 2022, 22, 1373. https:// doi.org/10.3390/s22041373

Academic Editor: Hyoungsik Nam

Received: 19 December 2021

Accepted: 27 January 2022

Published: 11 February 2022

Publisher's Note: MDPI stays neutral with regard to jurisdictional claims in published maps and institutional affiliations.

Copyright: (C) 2022 by the authors. Licensee MDPI, Basel, Switzerland. This article is an open access article distributed under the terms and conditions of the Creative Commons Attribution (CC BY) license (https:// creativecommons.org/licenses/by/ $4.0 /$ )
Abstract: Human activity recognition (HAR) using wearable sensors is an increasingly active research topic in machine learning, aided in part by the ready availability of detailed motion capture data from smartphones, fitness trackers, and smartwatches. The goal of HAR is to use such devices to assist users in their daily lives in application areas such as healthcare, physical therapy, and fitness. One of the main challenges for HAR, particularly when using supervised learning methods, is obtaining balanced data for algorithm optimisation and testing. As people perform some activities more than others (e.g., walk more than run), HAR datasets are typically imbalanced. The lack of dataset representation from minority classes hinders the ability of HAR classifiers to sufficiently capture new instances of those activities. We introduce three novel hybrid sampling strategies to generate more diverse synthetic samples to overcome the class imbalance problem. The first strategy, which we call the distance-based method (DBM), combines Synthetic Minority Oversampling Techniques (SMOTE) with Random_SMOTE, both of which are built around the k-nearest neighbors (KNN). The second technique, referred to as the noise detection-based method (NDBM), combines SMOTE Tomek links (SMOTE_Tomeklinks) and the modified synthetic minority oversampling technique (MSMOTE). The third approach, which we call the cluster-based method (CBM), combines Cluster-Based Synthetic Oversampling (CBSO) and Proximity Weighted Synthetic Oversampling Technique (ProWSyn). We compare the performance of the proposed hybrid methods to the individual constituent methods and baseline using accelerometer data from three commonly used benchmark datasets. We show that DBM, NDBM, and CBM reduce the impact of class imbalance and enhance $F 1$ scores by a range of 9-20 percentage point compared to their constituent sampling methods. CBM performs significantly better than the others under a Friedman test, however, DBM has lower computational requirements.

Keywords: activity recognition; wearable sensors; imbalanced activities; sampling methods

\section{Introduction}

Human activity recognition (HAR) using body-worn or wearable sensors is an active research topic in mobile and ubiquitous computing [1]. Activity recognition is a useful tool because it provides information on an individual's behaviour that enables computing systems not only to monitor but also to analyse and assist with a range of day-to-day tasks $[2,3]$.

Most HAR studies adopt a supervised learning approach [4]. Supervised learning typically requires immense amounts of labelled sensor data in order to train [2]. For such models to work well, the data are ideally recorded from a variety of real-word situations. Additionally, a diversity of sensor modalities and placements can help improve recognition performance $[5,6]$.

Sensor data obtained from real-life settings is typically of poor quality (noisy) and frequently has missing data [7]. These issues arise due to factors such as bad or faulty 
placement of sensors, or sensor malfunctioning [8]. Similarly, sensor data may often be highly imbalanced due to significant individual variations, with limited labels for certain activities [9]. Further barriers to obtaining sufficient quantities of real-world data include the prohibitive cost of devices, issues related to privacy, or a desire to reduce battery consumption [10]. Sensor data from certain activities can be difficult to obtain because of the rare but critical nature of those activities, such as falls in the elderly [2] or heart failure [11].

For HAR to succeed as a viable technique, there is an urgent need for new approaches at making up for this shortfall in critical and underrepresented real-world data [2,12]. An important part of this is solving the class imbalance problem [13]. Imbalance can occur in both the between-class distribution and as within-class imbalance [14]. Betweenclass imbalance occurs, for example, when some activities are performed less often than others [15]. As a result, the sample sizes for these activities are smaller, so a supervised model might not have enough data to learn adequately. The related concept of withinclass imbalance occurs when the same activity is performed in different ways by the same individual, yet there are insufficient examples from some of these for the model to generalise adequately [12].

There are usually two methods to solve class imbalance: data level (sampling) methods and algorithm level methods [16]. The data level approach involves changing a training set's class distribution by resampling. This might mean oversampling the minority classes, undersampling the majority classes, or a combination of both [17]. The algorithm level approach involves adjusting existing learning algorithms to focus more on the minority classes [16]. In this work we use the data level approach, which is less complex to configure and can be integrated with any learning algorithm.

The main contributions of the work are the following.

- We evaluate six sampling methods (SMOTE, Random_SMOTE, SMOTE_Tomeklinks, MSMOTE, CBSO, and ProWSyn) as solutions to the class imbalance problem across three commonly used datasets.

- We introduce three novel hybrid sampling approaches and show how these build on and improve upon their constituent methods. These are (1) DBM, a distance-based method that combines SMOTE and Random_SMOTE, (2) NDBM, a noise detectionbased method that combines SMOTE_Tomeklinks and MSMOTE, and (3) CBM, a cluster-based method that combines CBSO and ProWSyn.

- We compare how useful the sampling methods are to improve the learning from imbalanced human activity data using both shallow and deep machine learning algorithms. Specifically, we test KNN, Logistic regression (LR), Random Forest (RF) and Support Vector Machine (SVM), and a Multilayer perceptron (MLP) [18,19]. We show that the sampling methods are only useful to improve the performance of the MLP compared to the other classifiers for imbalanced human activity data.

The remainder of the paper is organised as follows. Section 2, shows some of the existing work on class imbalance problem and techniques to deal with it in HAR. Section 3 provides background on the sampling methods used. Section 3.4 introduces the proposed method and Section 4 describes the datasets. Section 5 describes the data analysis and experimental setup. Section 6 introduces the experimental results, and Section 7 discusses the findings from these.

\section{Related Work}

Several authors have highlighted the importance of the class imbalance problem in HAR $[2,12,20,21]$. Ni et al. introduced a HAR system based on using the stacked denoising autoencoder (SDAE) to recognise static and dynamic ambulatory activities, such as standing and running, using accelerometers and gyroscopes [22]. The performance of their model dropped as the class (activity) distribution of samples became unbalanced. They used sampling techniques such as SMOTE and random undersampling to tackle the problem. Based on their experimental result, the sampling techniques were more successful than 
random undersampling at successfully treating imbalance and improving recognition performance. This is because the indiscriminate nature of the undersampling approach can lead to useful data being discarded.

Despite the promising results, $\mathrm{Ni}$ et al's. work is limited in that it oversamples the entire dataset. Implementing oversampling before splitting a dataset into different train and test partitions can result in information leakage from the original test data to the newly produced training data and this can then lead to overly optimistic classification performance [23]. In other words, the learning algorithm's performance might be less about its ability to generalise to the test data appropriately, than it is an indication of similar patterns in both train and test data due to information leakage. In the current work we avoid the information leakage problem by sampling exclusively on the training set.

Chen et al. [20] used data from accelerometers and gyroscopes to recognise activities such as walking, jogging, and jumping - again using an imbalanced dataset. They reported that the classifier always showed a good performance in recognising the majority class, whereas its performance was inadequate for the minority classes. Again, SMOTE was used to increase the count of underrepresented activities, leading to improved overall performance. One limitation of this work was that it only evaluated a single oversampling method. In contrast, our work evaluates a combination of different methods.

Inspired by the concept of data fusion, we introduce three hybrid sampling methodsDBM, NDBM, and CBM - which combine the outputs from different sampling methods. Fusion of diverse data sources and sensor modalities is a widely explored approach for improving recognition performance in HAR (e.g., [5,18,24,25]). Similarly, fusion of multiple, diverse, weak learners to produce a strong ensemble is a well-studied and effective approach in machine learning [26]. We hypothesise that by combining outputs from different sampling strategies we diversify the synthetic data and in turn improve the generalization ability of our learning models.

\section{Sampling Methods}

The underlying sampling methods used in this work can be categorised into three types: distance-based, noise detection-based, and cluster-based.

\subsection{Distance-Based}

SMOTE and Random_SMOTE both use distance-based algorithms to oversample the training data. SMOTE [27] takes an instance of the minority class $x$ from the training set, and then computes its $K$ nearest neighbours, identified as the shortest Euclidean distances between itself and other instances of the same class. To produce a synthetic sample, $x_{\text {new }}$, SMOTE randomly selects the $K$ nearest neighbours from the minority class, e.g., $x_{k}$ for the $k$ th nearest neighbour, and computes the difference $x_{k}-x$. The new synthetic sample, $x_{\text {new }}$, is computed by multiplying this difference by a random number between 0 and 1 using Equation (1). The new synthetic instance $x_{\text {new }}$ will lie along the line between $x$ and $x_{k}$ :

$$
x_{\text {new }}=x+|| x-x_{k}|| \times \text { rand }(0,1)
$$

Unfortunately, the linear design of SMOTE can lead to overfitting. Random_SMOTE [28] tackles this by opening up a much wider region for oversampling. For each minority instance, $x$, two minority samples $x_{1}$ and $x_{2}$ (with $x_{1,2} \neq x$ ) are randomly selected. A temporary synthetic sample $x_{t m p}$ is then generated along the line between $x_{1}$ and $x_{2}$, as shown in Equation (2):

$$
x_{t m p}=x_{1}+\left\|x_{2}-x_{1}\right\| \times \text { rand }(0,1)
$$

The final synthetic sample $x_{\text {new }}$ is then created along the line between $x_{t m p}$ and the original sample $x$ using Equation (3):

$$
x_{\text {new }}=x+\left\|x_{\text {tmp }}-x\right\| \times \text { rand }(0,1)
$$




\subsection{Noise Detection-Based}

Real world data contain noise from a variety of sources that can lead to poor recognition performance [29,30]. Frenay et al. [31] indicated that class noise (also known as label noise) is one of the most harmful noises in machine learning. This kind of noise can occur, for example, if a minority class sample is incorrectly labelled with a majority class label [16]. The SMOTE-Tomek Link [32] and modified synthetic minority oversampling technique (MSMOTE) algorithms are specifically designed to detect this kind of noise in order to minimize the risk of creating noisy synthetic samples [33].

SMOTE-Tomek Links oversamples using SMOTE on top of a Tomek link data cleaning step [32]. Tomek link works as follows: Consider two samples $x_{a}$ and $x_{b}$ belonging to different classes, where $d\left(x_{a}, x_{b}\right)$ is the Euclidean distance between $x_{a}$ and $x_{b}$. A Tomek link is identified as an $\left(x_{a} x_{b}\right)$ pair if there is no sample $z$ that meets the following conditions: $d\left(x_{a}, z\right)<d\left(x_{a}, x_{b}\right)$ or $d\left(x_{b}, z\right)<d\left(x_{a}, x_{b}\right)$. That is, $x_{a}$ and $x_{b}$ are each other's nearest neighbours [34]. Tomek links are therefore likely to be comprised of either boundary samples or noisy samples $[30,35,36]$. SMOTE-Tomek Links generates synthetic data in two steps [32]. First, the original minority training data are oversampled using SMOTE. Second, Tomek links are identified in the training data and removed to rebalance the data set.

MSMOTE is an improved version of SMOTE which first uses KNN to assign minority samples into three types: safe, border, and noise [33]. If a minority labelled sample is the same as the labels of its $k$ near neighbours, then the sample is defined as 'safe'. If the labels are all different, then the sample is identified as 'noise'. Finally, if the sample is neither safe nor noise, it is classed as a 'border' sample. The second step of MSMOTE uses SMOTE to generate new samples. However, the random selection of neighbours is different depending on whether the sample is safe, border, or noise. For safe samples, MSMOTE will randomly choose the $K$ nearest neighbours. For border samples, the algorithm only selects the nearest neighbour (i.e., $K=1$ ). Noise samples are simply disregarded.

\subsection{Cluster-Based}

The cluster-based sampling methods include Cluster-Based Synthetic Oversampling (CBSO) and Proximity Weighted Synthetic Oversampling Technique (ProWSyn).

CBSO integrate clustering and SMOTE-it uses agglomerative clustering to first cluster minority samples with the aim of identifying those minority samples which are close to the majority samples border [37]. CBSO produces samples only in the neighbourhood of minority samples that are close to majority neighbours using SMOTE. For instance, in order to produce a new sample, CBSO will select a sample $x$ from the minority class and randomly choose a minority sample $x_{k}$ from $x^{\prime}$ s cluster (using SMOTE Equation (1)), to produce a new sample.

ProWSyn is another cluster-based sampling method [38]. This algorithm computes the distance between minority class samples and majority class samples in order to assign greater weights to the minority samples. These weights are then used to assign greater significance to the minority samples during learning. ProWSyn operates in two steps: The first step splits the minority data into partitions $(P)$ according to their distance from the class boundary. ProWSyn assigns a proximity level $(L)$ to each partition. The level increases with distance from the boundary. When minority class samples are assigned to lower proximity levels, then they are considered more important for learning because they are close to the boundary. However, in cases where they are assigned higher proximity levels they are considered less important [38].

\subsection{Proposed Hybrid Methods}

All three proposed hybrid approaches, DBM, NDBM, and CBM, concatenate synthesized training data obtained from the constituent sampling methods. DBM combines SMOTE and Random_SMOTE. NDBM combines SMOTE_Tomeklinks and MSMOTE. Finally, CBM combines CBSO and ProWSyn. The three methods are evaluated as shown in Figure 1. Taking $D$ as the original dataset, we first split $D$ into $D_{\text {train }}$ and $D_{\text {test }}$. $D_{\text {train }}$ is 
then oversampled using the constituent methods. For more clarification, lets refer to the $D_{\text {train }}$ as $d$ and, for instance, if $D B M$ is used to oversample $d$, it will be:

$$
d_{D B M}=\operatorname{SMOTE}\left(D_{\text {train }}, \alpha\right)+\text { Random_SMOTE }\left(D_{\text {train }}, \alpha\right)
$$

where $\alpha$ is the oversampling ratio. In case of using NDBM, it is denoted as:

$$
d_{\text {NDBM }}=\text { SMOTE_TomekLinks }\left(D_{\text {train }}, \alpha\right)+\operatorname{MSMOTE}\left(D_{\text {train }}, \alpha\right)
$$

For the CBM, it is referred to as:

$$
d_{C B M}=\operatorname{ProWSyn}\left(D_{\text {train }}, \alpha\right)+C B S O\left(D_{\text {train }}, \alpha\right)
$$

We then concatenate the synthesized data to increase the size of $D_{\text {train }}$. The oversampled $D_{\text {train }}$ is used to train a classifier, which is then evaluated on the left-aside $D_{\text {test }}$.

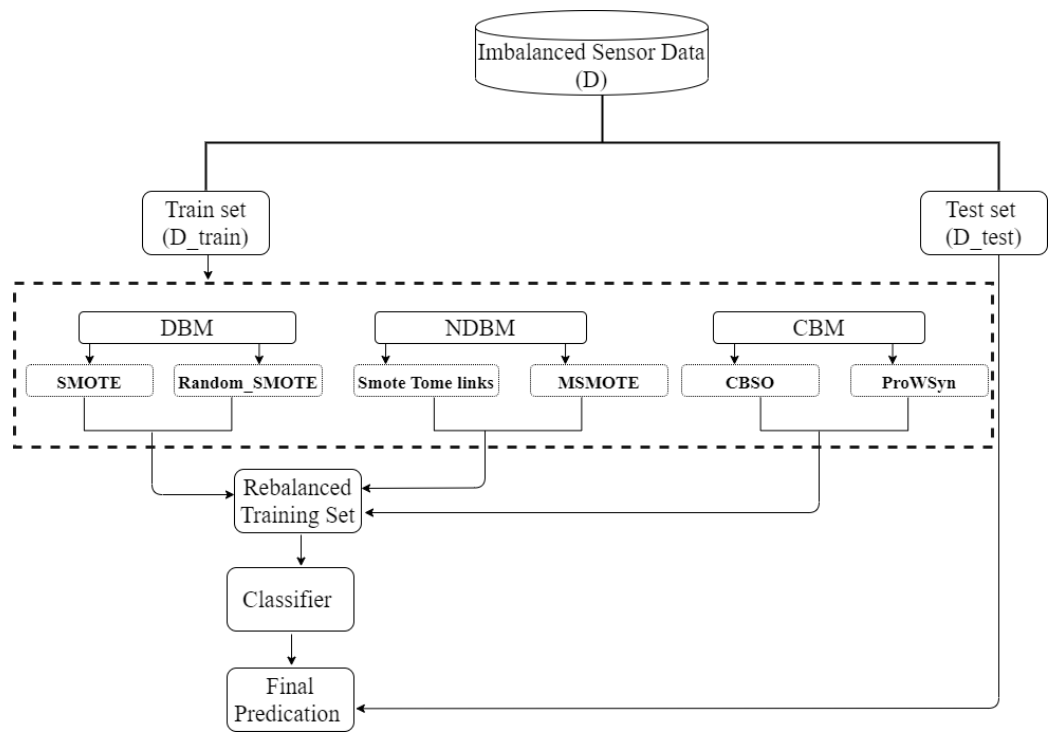

Figure 1. Overview of the process used for splitting, oversampling, and evaluating the data.

\section{Datasets}

We use three datasets that are widely used by HAR researchers: Opportunity [39], Physical Activity Monitoring (PAMAP2) [40], and Activities of Daily Living (ADL) [41] Each of these comprise many individuals performing different types of human activity, including ambulation and daily living activities [42]. The ambulation activities are typically performed over a longer period of time, which comes in two difference forms: static (less repetitive) such as standing, or dynamic (more repetitive), for example, running. Shoaib et al. [43] describe these activities as 'simple' because they might be easily identified using a wrist-worn accelerometer placed at an individual wrist. Daily activities might consist of hand gestures such as waving hands or hand-to-mouth gestures (HMG), for example, eating or drinking [44]. Daily activities are not as repetitive as ambulatory dynamic activities, and these daily activities often are concurrent with each other due to their similar gestures such as eating, drinking, and brushing teeth [45]. Such activities are referred to as 'complex' because they are more challenging to identify using a single accelerometer compared to simple activities [43].

\subsection{Opportunity}

The Opportunity dataset was collected from 72 sensors, with different types of sensors integrated into the environment, objects and worn on participants' bodies [39]. Four participants performed daily living scenarios in a simulated kitchen environment. The dataset included around $6 \mathrm{~h}$ of recordings and was sampled at $30 \mathrm{~Hz}$. The activities were 
annotated on two different levels: locomotion and gesture. For example, cleaning up and open door were labelled as gestures, with sitting and lying making up the locomotion subset. Here we focus solely on gesture activities. Figure 2 shows 17 activities categorised as gestures, including Open Door1, Open Door2, Close Door1, Close Door2, Open Fridge, Close Fridge, Open Dishwasher, Close Dishwasher, Open Drawer1, Close Drawer1, Open Drawer2, Close Drawer2, Open Drawer3, Close Drawer3, Clean Table, Drink from Cup, and Toggle Switch. The dataset contains several on-body and object sensors, but in this paper we use only the accelerometer in the lower right arm-worn inertial measurement unit (IMU).

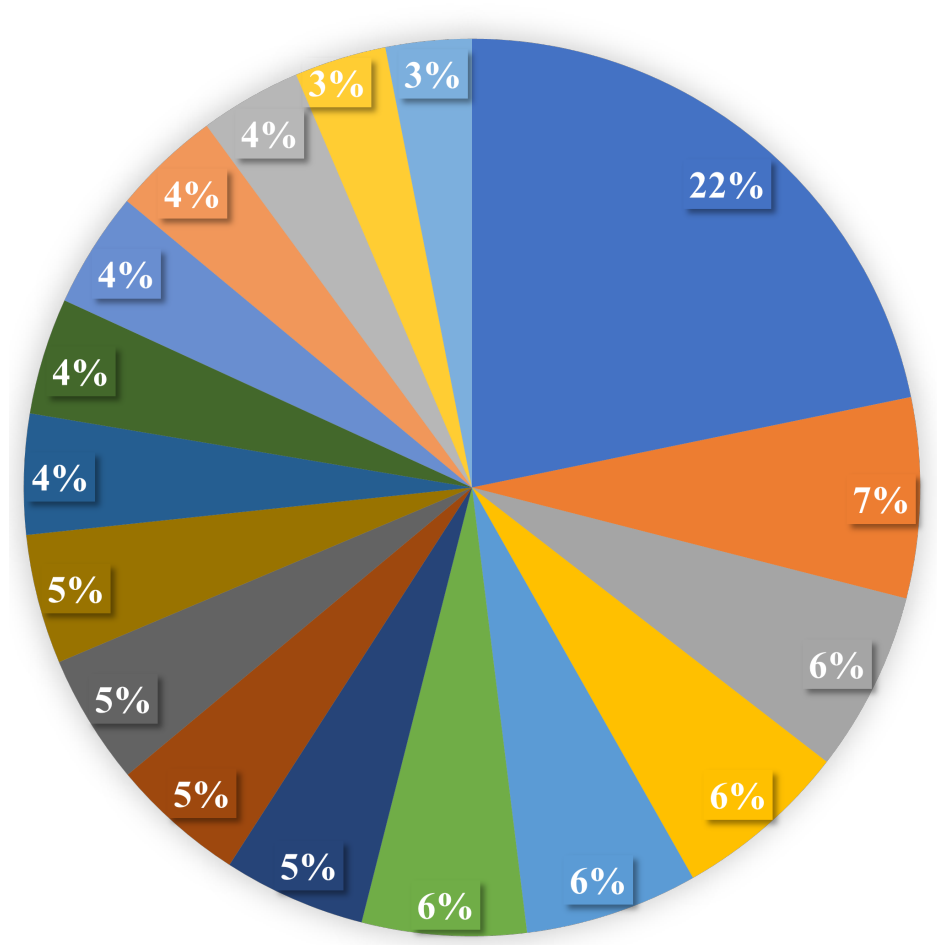

- Drink_from_Cup
- Clean_Table
Close_Door2
Close_Door1
- Open_Door2
- Open_Door1
- Open_Dishwasher
- Toggle_Switch
- Open_Fridge
-Close_Dishwasher
-Close_Fridge
-Open_Drawer3
-Close_Drawer_3
Open_Drawer1
Open_Drawer2
Close_Drawer2
Close_Drawer1

Figure 2. Activity distribution of the Opportunity dataset.

\subsection{PAMAP2}

The Physical Activity Monitoring dataset (PAMAP2) was collected from 9 participants who performed 12 activities for over $10 \mathrm{~h}$ and it was sampled at $100 \mathrm{~Hz}$. Data were recorded by using IMUs placed on the hand, chest, and ankle [40]. Here we use only the accelerometer sensor of the hand-worn IMU. Figure 3 shows the activity distribution, and it can be seen that the dataset is imbalanced. It contains both simple and sporting activities such as walking, running, cycling, Nordic walking, and rope jumping. It also includes posture activities such as lying, sitting, and standing. Activities of daily living (ascending stairs, descending stairs), and households activities such as vacuum cleaning and ironing are also included.

\section{3. $A D L$}

The Activities of Daily Living (ADL) dataset is a public dataset collected using a single chest-worn wearable accelerometer on 15 participants [41]. The sampling rate of the accelerometer was $52 \mathrm{~Hz}$. The participants performed seven daily living activities. The activities include Working at Computer (WAC), Standing Up, Walking and Going Up/Downstairs (SWGUDS), Standing, Walking, Going Up/Downstairs (GUDS), Walking and Talking with Someone (WATWS), and Talking while Standing (TWS). Figure 4 shows the activities distribution of the ADL dataset which indicates that the dataset is imbalanced. 




Figure 3. Activity distribution of the PAMAP2 dataset.

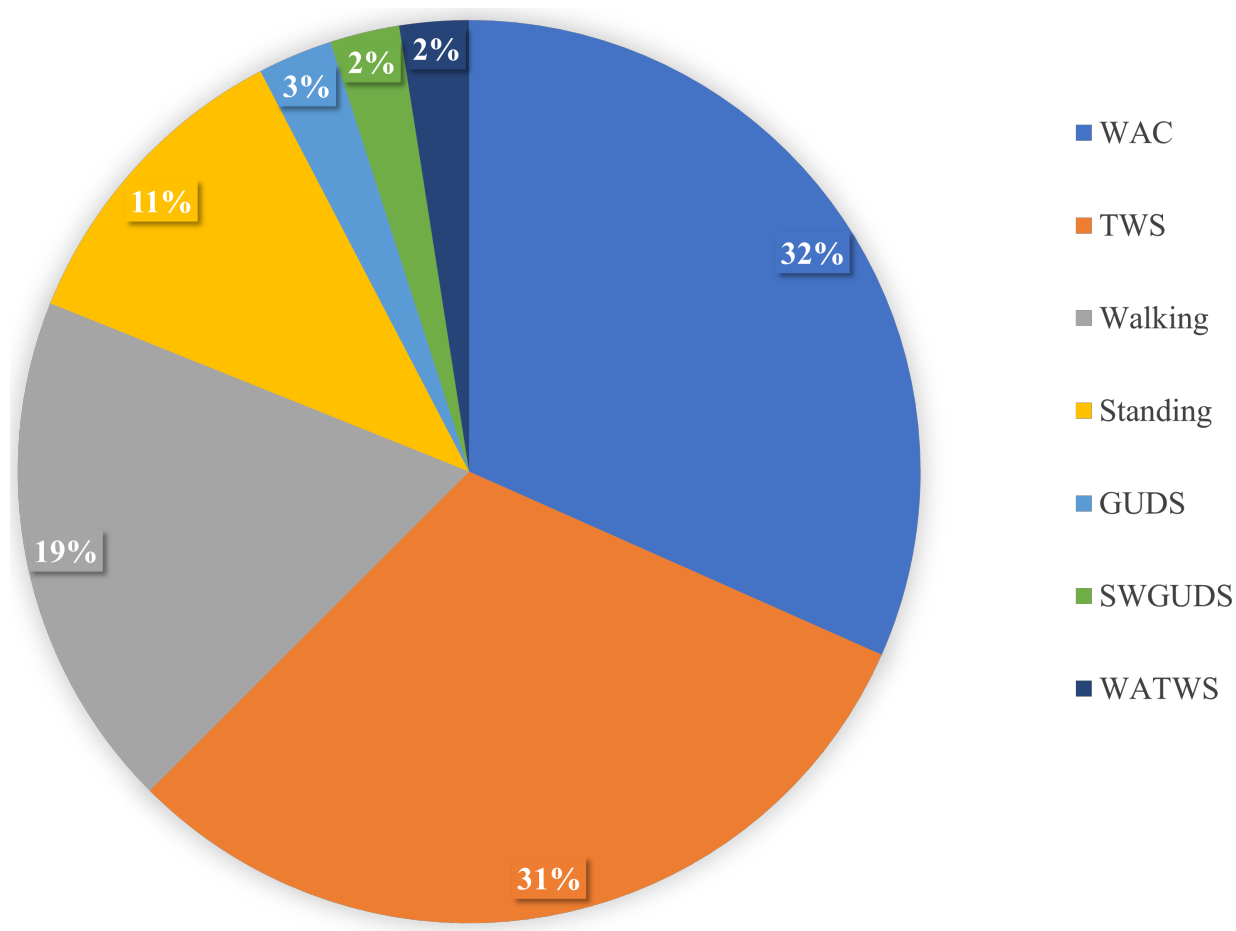

Figure 4. Activity distribution of the ADL dataset.

\section{Data Analysis}

\subsection{Data Preprocessing}

We explore how our proposed sampling methods might enhance a human activity model's performance in a real-life scenario where only a single 3-axis accelerometer is available. Although recognition performance is typically better when multiple sensors are used, in many scenarios access to multiple sensors is limited (e.g., in a single wrist-worn device) [10]. 
As a pre-processing step, we first calculate the Euclidean norm $\left(\sqrt{x^{2}+y^{2}+z^{2}}\right)$ of each 3-axis sensor to ensure the data are invariant to shifting orientation of the sensors [46]. We then apply a non-overlapping sliding window to segment the data [24]. Table 1 provides more details such as the number of subjects, sampling rate, the window size, and sensor position we use.

We extract six time-domain features including mean, standard deviation, minimum, maximum, median, and range. The selected features are highlighted further in Table 2. These features are efficient as well as fast to compute [18].

Table 1. Datasets details.

\begin{tabular}{cccccc}
\hline Dataset & $\begin{array}{c}\text { Number of } \\
\text { Subjects }\end{array}$ & $\begin{array}{c}\text { Sample } \\
\text { Rate }\end{array}$ & Window Size (s) & Sensor Position & $\begin{array}{c}\text { Number of } \\
\text { Sensors Used }\end{array}$ \\
\hline Opportunity & 4 & 32 & 2 & Right Arm & 1 accelerometer \\
PAMAP2 & 8 & 100 & 3 & Dominant Wrist & 1 accelerometer \\
ADL & 15 & 52 & 10 & Chest & 1 accelerometer \\
\hline
\end{tabular}

Table 2. Features description $[18,43]$.

\begin{tabular}{cc}
\hline Feature & Description \\
\hline Mean & It provides the average value of sensor data within a segment \\
\hline Standard deviation & It describes how much sensor data are spread around the mean \\
\hline Minimum & The minimum value of sensor data within a segment \\
\hline Maximum & The maximum value of sensor data within a segment \\
\hline Median & It finds the middle number of a sample within a segment \\
\hline Range & The difference between the maximum and the minimum of sensor data within a segment
\end{tabular}

\subsection{Parameters Setting}

All of the evaluations in this work were carried out using a collection of shallow learning methods-specifically, SVM, LR, kNN, and RF-as well as a deep learning method based on MLP. The parameters for SVM, LR, and KNN were found using grid search (see Appendix C for details). For RF and MLP, we use the default settings provided by the Python implementation [47]. The MLP architecture that was used in the experiments is presented in Table 3.

Table 3. MLP architecture details.

\begin{tabular}{ccccccc}
\hline $\begin{array}{c}\text { Hidden } \\
\text { Layers }\end{array}$ & $\begin{array}{c}\text { Activation } \\
\text { Function }\end{array}$ & Optimizer & $\begin{array}{c}\text { Loss } \\
\text { Function }\end{array}$ & $\begin{array}{c}\text { Learning } \\
\text { Rate }\end{array}$ & Regularization & Epochs \\
\hline 100 & Relu & Adam & Cross-entropy & 0.001 & L2 penalty & 200 \\
\hline
\end{tabular}

Note that for brevity we include only the full results related to the overall bestperforming classifier, MLP. The main findings using the remaining classifiers were broadly in agreement (as will be shown in Section 6.7). The full results for the remaining four classifiers are provided in Appendix B.

We also use the default settings on the Python implementation of our sampling algorithms_SMOTE, Random_SMOTE, SMOTE_TomekLinks, MSMOTE, CBSO, and ProWSyn. Number of neighbours and the number of samples to generate are common parameters among all sampling methods. In addition, other sampling methods use specific parameters, for example, ProWSyn utilizes number of levels. Ref. [37] provides more details about these parameters.

The percentage of samples to be created by a sampling method was set to $100 \%$, which means that the number of minority samples in the training set will be equal to the number of majority samples in the training set after sampling. 


\subsection{Evaluation Method}

Performance is measured using weighted F1 score, recall, and precision [12]. Precision records the proportion of class predictions that are correct, whereas Recall records the proportion of actual class samples that are correct [48]. The weighted F1 score used here weighs classes based on their sample proportion and is calculated as (e.g., [1]):

$$
\text { F1 score }=\sum_{i} 2 * w_{i} \frac{\times \text { Precision }_{i} \times \text { Recall }_{i}}{\text { Precision }_{i}+\text { Recall }_{i}}
$$

Here, $i$ corresponds to the class. $w_{i}=n_{i} / N$ corresponds to the proportion of class $i$ and $n_{i}$ is the number of samples of the class $i . N$ is the total number of samples.

Pirttikangas et al. [49] suggested to combine all the data from different subject into one dataset. They augured this was because of the individual variation in body worn acceleration which is often dominates by strong commonalities among individuals in activity patterns.

Consequently, we followed Pirttikangas et al.'s [49] suggestion in this work and used 3-fold cross-validation to train the parameters in our analyses. We did not use more than 3 folds as most of the activities have a very low number of samples in some datasets. As an additional measure of reliability, our evaluations are all repeated 30 times using different random selections of data. The final results are presented as the mean (and standard deviation) of the F1 score over these repetitions. In the future we aim to use a leave-one-subject-out approach.

ANOVA and Friedman statistical tests were performed to discover whether there are significant differences in performance between the sampling methods across the five classifiers [50,51].

\section{Results}

Here we present the final results of each of the sampling methods and our hybrid methods vs the baseline (no resampling) case for each of the three datasets. We also present an evaluation of the power considerations for each method.

\subsection{Distance-Based Method (DBM)}

Table 4 shows the main results for the MLP classifier using DBM versus its constituent methods, SMOTE, and Random_SMOTE. The first thing to notice is a universal improvement when sampling is used compared to the baseline.

On the ADL dataset, the DBM F1 score is $92.59 \%$, a 5.39 percentage point (pp) improvement over baseline, a $0.35 \%$ improvement over SMOTE, and a 1.52 pp improvement over Random_SMOTE. On Opportunity, DBM's F1 score is low (48.49\%), however, this is a large 19.64 pp improvement on the baseline, and a 5 pp improvement over both constituent sampling methods. On PAMAP2, DBM F1 score is $80.15 \%$, which is an 8.3 pp improvement on the baseline, and 5 pp on both constituent methods. 
Table 4. Distance-based method results. Comparing the performance of MLP on DBM, SMOTE, and Random_SMOTE for multiple datasets. The reported mean of $F 1$ score and ( \pm standard deviation), recall, and precision are obtained from 30 repetitions. The $F 1$ score, recall, and precision are in \%. Highest scores are shown in bold.

\begin{tabular}{ccccc}
\hline Data & Method & F1 Score & Recall & Precision \\
\hline ADL & Baseline & $87.2( \pm 0.047)$ & 87.03 & 89.02 \\
& SMOTE & $92.24( \pm 0.069)$ & 91.44 & 94.21 \\
& Random_SMOTE & $91.07( \pm 0.086)$ & 90.31 & 93.22 \\
Opportunity & DBM & $\mathbf{9 2 . 5 9}( \pm 0.081)$ & $\mathbf{9 1 . 9}$ & $\mathbf{9 4 . 2 6}$ \\
& Baseline & $28.85( \pm 0.017)$ & 34.1 & 45.57 \\
& SMOTE & $42.95( \pm 0.043)$ & 42.45 & 45.75 \\
PAMAP2 & Random_SMOTE & $42.74( \pm 0.04)$ & 42.19 & $\mathbf{5 0 . 6 3}$ \\
& DBM & $\mathbf{4 8 . 4 9}( \pm 0.052)$ & $\mathbf{4 8 . 1 8}$ & $\mathbf{7 5 . 4 9}$ \\
& Baseline & $71.85( \pm 0.081)$ & 72.73 & 77.69 \\
& SMOTE & $74.73( \pm 0.055)$ & 74.64 & $\mathbf{8 1 . 9 3}$ \\
\hline
\end{tabular}

\subsection{Noise Detection-Based Method (NDBM)}

Table 5 demonstrates the MLP classifier performance of baseline, NDBM, SMOTE_TomekLinks, and MSMOTE across all datasets. Again, a large improvement is evident for all datasets when using sampling versus baseline.

On the ADL dataset, the NDBM F1 score is $93.7 \%$, a 5.39 pp improvement over the baseline, and between 1-2 pp improvement over the constituent sampling methods. On the Opportunity dataset, NDBM performance is low (with $F 1$ at $46.95 \%$ )-however, this is a dramatic $18.1 \mathrm{pp}$ improvement over the baseline case. On the PAMAP2 dataset, the NDBM F1 score is $79.43 \%$, a 7.58 pp improvement on the baseline, and 5 pp improvement over both constituent methods.

Table 5. Noise detection-based results. Comparing the performance of MLP for NDBM, MSMOTE, and SMOTE_TomekLinks on multiple datasets. The reported mean of $F 1$ score and ( \pm standard deviation), recall, and precision are obtained from 30 repetitions. The $F 1$ score, recall, and precision are in $\%$. Highest scores are shown in bold.

\begin{tabular}{ccccc}
\hline Data & Method & F1 Score & Recall & Precision \\
\hline ADL & Baseline & $87.2( \pm 0.047)$ & 87.03 & 89.02 \\
& SMOTE_TomekLinks & $91.41( \pm 0.071)$ & 90.52 & 93.56 \\
& MSMOTE & $90.7( \pm 0.067)$ & 89.65 & 92.66 \\
Opportunity & NDBM & $\mathbf{9 2 . 7}( \pm 0.065)$ & $\mathbf{9 1 . 6 9}$ & $\mathbf{9 4 . 7 7}$ \\
& Baseline & $28.85( \pm 0.017)$ & 34.1 & 49.57 \\
& SMOTE_TomekLinks & $42.89( \pm 0.039)$ & 43.15 & 42.07 \\
PAMAP2 & MSMOTE & $39.71( \pm 0.074)$ & 39.58 & $\mathbf{4 8 . 8 6}$ \\
& NDBM & $\mathbf{4 6 . 9 5}( \pm 0.067)$ & $\mathbf{4 6 . 9 7}$ & 75.49 \\
& Baseline & $71.85( \pm 0.081)$ & 72.73 & 77.13 \\
& SMOTE_TomekLinks & $74.24( \pm 0.054)$ & 74.51 & $\mathbf{7 3 . 7 8}$ \\
\hline
\end{tabular}

\subsection{Cluster-Based Method (CBM)}

Table 6 demonstrates the MLP classifier performance of baseline, CBM, CBSO, and ProWSyn across all datasets. Again, a clear improvement is evident for all datasets when using sampling versus baseline.

On the ADL dataset, the CBM F1 score is $92.96 \%$, a 5.76 pp improvement over the baseline, and a 1.8-1.4 pp improvement over the constituent sampling methods. On the Opportunity dataset, CBM performance is low (with $F 1$ at $48.87 \%$ ) - however, this is a dramatic 20.02 pp improvement over the baseline case. On the PAMAP2 dataset, the CBM F1 score is $81.15 \%$, a 9.13 pp improvement over the baseline, and a 5.29 pp and 6.56 pp improvement over CBSO and ProWSync, respectively. 
Table 6. Cluster-based results. Comparing the performance of MLP using CBM, CBSO, and ProWsyn on multiple datasets. The reported mean of $F 1$ scores and ( \pm standard deviation), recall, and precision are obtained from 30 repetitions. The $F 1$ score, recall, and precision are in \%. Highest scores are shown in bold.

\begin{tabular}{ccccc}
\hline Data & Method & F1 Score & Recall & Precision \\
\hline ADL & Baseline & $87.2( \pm 0.047)$ & 87.03 & 89.02 \\
& CBSO & $91.16( \pm 0.09)$ & 90.22 & 93.66 \\
& ProWSyn & $91.56( \pm 0.091)$ & 90.98 & 93.7 \\
Opportunity & CBM & $\mathbf{9 2 . 9 6}(0.087)$ & $\mathbf{9 1 . 9 3}$ & $\mathbf{9 5 . 2 9}$ \\
& Baseline & $28.85( \pm 0.017)$ & 34.1 & 29.57 \\
& CBSO & $42.92( \pm 0.023)$ & 42.96 & 45.12 \\
PAMAP2 & ProWSyn & $42.78( \pm 0.055)$ & 43.47 & $\mathbf{4 0 . 6 7}$ \\
& CBM & $48.87( \pm 0.045)$ & 72.82 & 75.49 \\
& Baseline & $71.85( \pm 0.081)$ & 75.43 & 77.5 \\
& CBSO & $75.69( \pm 0.042)$ & 74.4 & $\mathbf{8 2 . 5 4}$ \\
\hline
\end{tabular}

6.4. Comparing the Performance of the Proposed Sampling Approaches DBM, NDBM, and CBM

Table 7 combines the headline results from our proposed hybrid methods. On the ADL dataset, CBM outperforms the others, with an F1 score of 92.96\%. On Opportunity, CBM (48.87\% F1) narrowly outperforms DBM (48.49\% F1) and NDBM (46.95\% F1). Similarly, on PAMAP2, CBM (80.98\% F1) outperforms DBM (80.15\% F1) and NDBM (79.43\% F1). The standard deviation across recognition scores for all evaluations is low throughout, with the maximum deviation being no more than 0.087 .

Table 7. Comparing performance of DBM, NDBM, and CBM on multiple datasets. The reported mean of $F 1$ scores and ( \pm standard deviation), recall, and precision were obtained from 30 repetitions. The F1 score, recall, and precision are in \%. Highest scores are shown in bold.

\begin{tabular}{ccccc}
\hline Data & Method & F1 Score & Recall & Precision \\
\hline ADL & Baseline & $87.2( \pm 0.047)$ & 87.03 & 89.02 \\
& DBM & $92.59( \pm 0.081)$ & 91.9 & 94.26 \\
& NDBM & $92.7( \pm 0.065)$ & 91.69 & 94.77 \\
Opportunity & CBM & $\mathbf{9 2 . 9 6}( \pm 0.087)$ & $\mathbf{9 1 . 9 3}$ & $\mathbf{9 5 . 2 9}$ \\
& Baseline & $28.85( \pm 0.017)$ & 34.1 & 50.63 \\
& DBM & $48.49( \pm 0.052)$ & 48.18 & 48.86 \\
PAMAP2 & NDBM & $46.95( \pm 0.067)$ & 46.97 & $\mathbf{5 0 . 6 7}$ \\
& CBM & $\mathbf{4 8 . 8 7}( \pm 0.045)$ & 72.82 & 81.93 \\
& Baseline & $71.85( \pm 0.081)$ & 80.23 & 81.35 \\
& DBM & $80.15( \pm 0.046)$ & 79.46 & $\mathbf{8 2 . 5 4}$ \\
\hline
\end{tabular}

\subsection{Results for Minority Activities}

For the Opportunity dataset, multiple activities were underrepresented, such as Open_Fridge, Open_Drawer3, and Close_Drawer3. Figure 5 indicates that the proposed DBM, NDBM, and CBM improve the F1 score of the MLP in recognising the underrepresented activities. Figure 5 also shows that without applying the sampling methods (baseline), the MLP classifier could not identify the Open_Fridge activity. By applying the proposed sampling methods, the MLP's ability to recognise underrepresented activities improved. For example, the $F 1$ of the MLP's ability to classify the Open_Fridge activity improved by more than 10 pp using the DBM, NDBM, and CBM. 


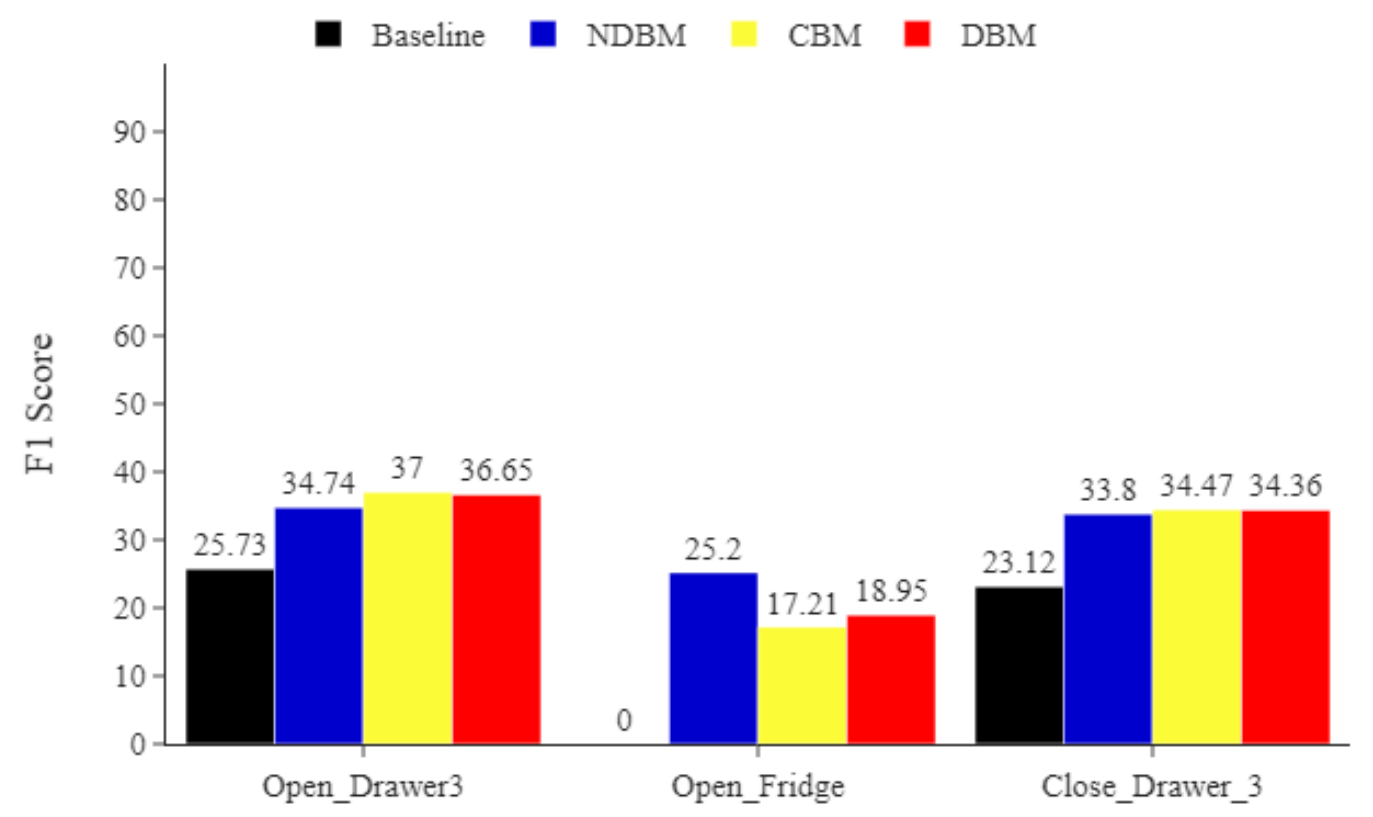

Figure 5. Opportunity minority classes. Comparing the impact of DBM, NDBM, and CBM on activity recognition performance, using MLP for the most underrepresented activities Open_Fridge, Open_Drawer3, and Close_Drawer3. The reported means of $F 1$ scores are obtained from 30 repetitions. The F1 score is in \%.

On the ADL dataset, Figure 6 also suggests that by applying the DBM, NDBM, and CBM MLP classifier, F1 score was improved by more than $10 \mathrm{pp}$ and gained a significant advantage in identifying the underrepresented activities, including Going Up/Downstairs (GUDS), Standing Up, Walking and Going Up/Downstairs (SWGUDS), and Walking and Talking with Someone (WATWS).

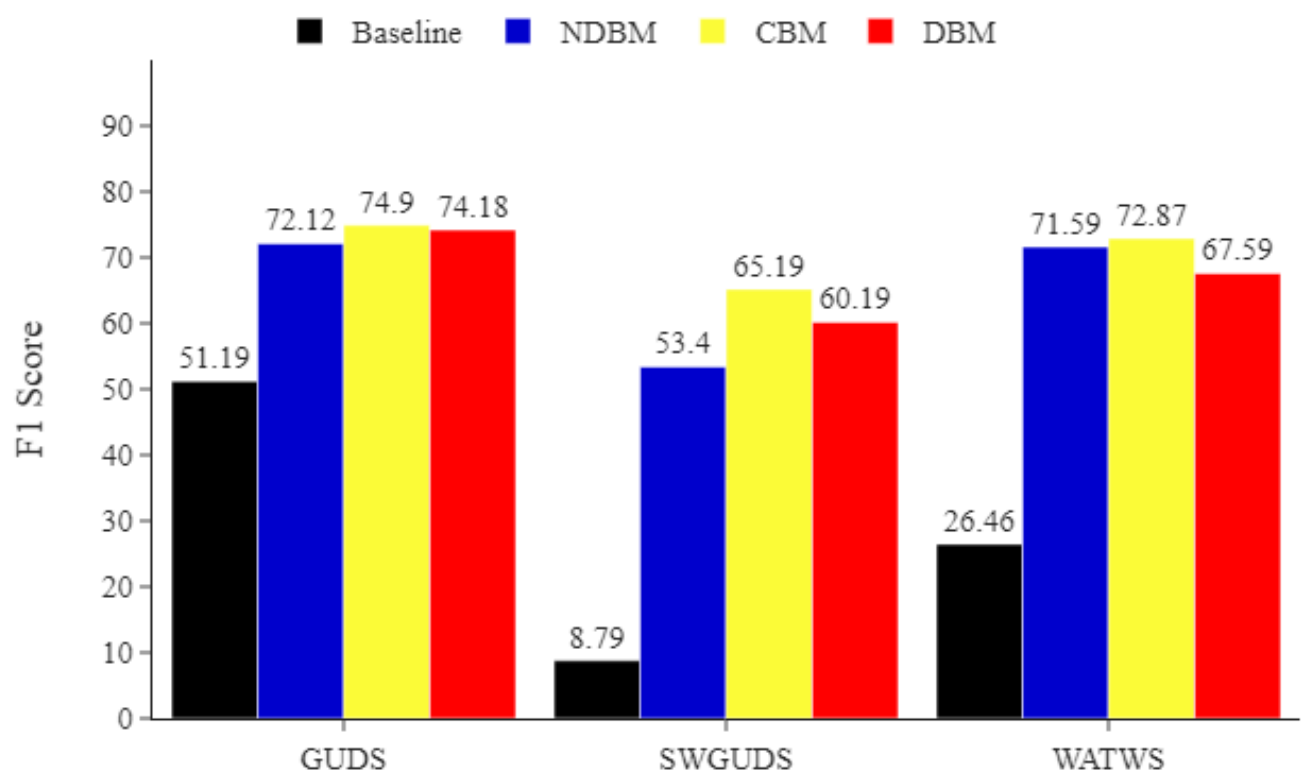

Figure 6. ADL minority classes. Comparing the impact of DBM, NDBM, and CBM on activity recognition performance, using MLP for the most underrepresented activities (Going Up/Downstairs (GUDS), Standing Up, Walking and Going Up/Downstairs (SWGUDS), and Walking and Talking with Someone (WATWS)). The reported means of $F 1$ scores are obtained from 30 repetitions. The $F 1$ score is in \%.

Similarly, on the PAMAP2 dataset, Figure 7 implies that the MLP classifier was more capable of identifying the underrepresented activities, including rope jumping, running, 
descending stairs, and ascending stairs, when the proposed DBM, NDBM, and CBM were used. For example, the performance of the MLP improved on the $F 1$ score by at least $4 \mathrm{pp}$ when identifying the underrepresented rope jumping activity.

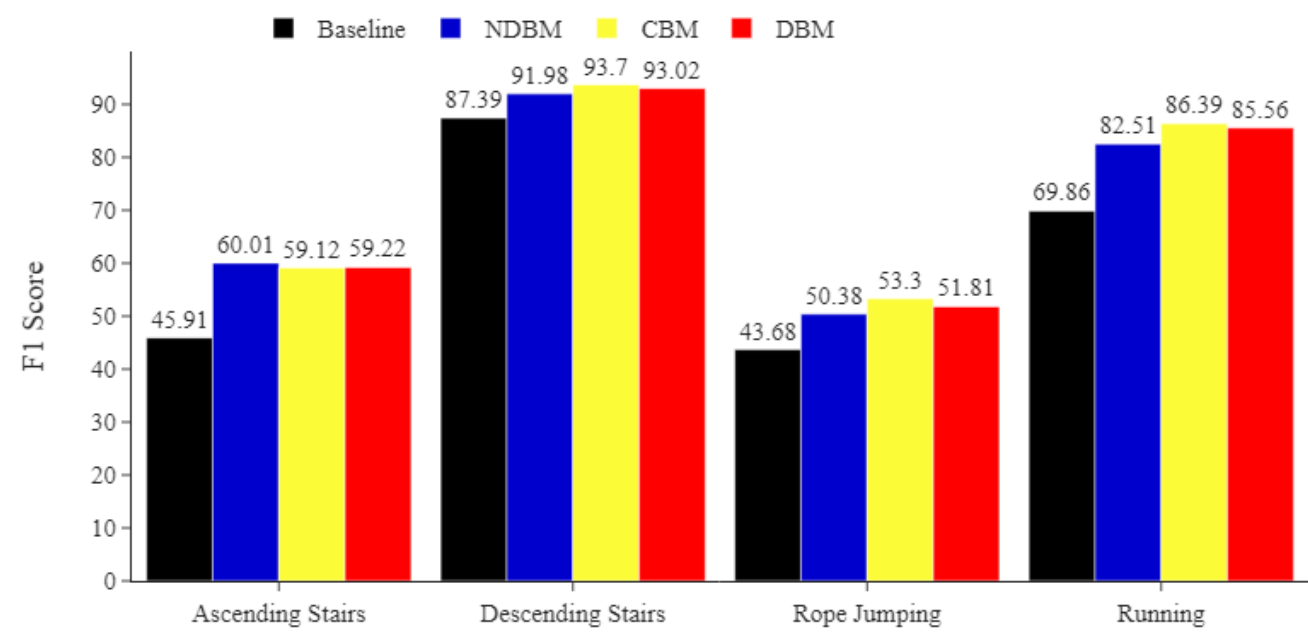

Figure 7. PAMAP2 minority classes. Comparing the impact of DBM, NDBM, and CBM on activity recognition performance, using MLP for the most underrepresented activities (ascending stairs, descending stairs, rope jumping, and running). The reported means of $F 1$ scores are obtained from 30 repetitions. The $F 1$ score is in \%.

\subsection{Run Times for DBM, NDBM, and CBM}

Figure 8 offers a comparison for each proposed sampling method in terms of run times. The analysis was performed on a Fierce PC with 16 GB RAM, Intel Core i7-7700 processor with $3.60 \mathrm{GHz}$ and using Ubuntu 16.04 LTS (64-bits). DBM demonstrated the best performance in terms of training time compared to NDBM and CBM.

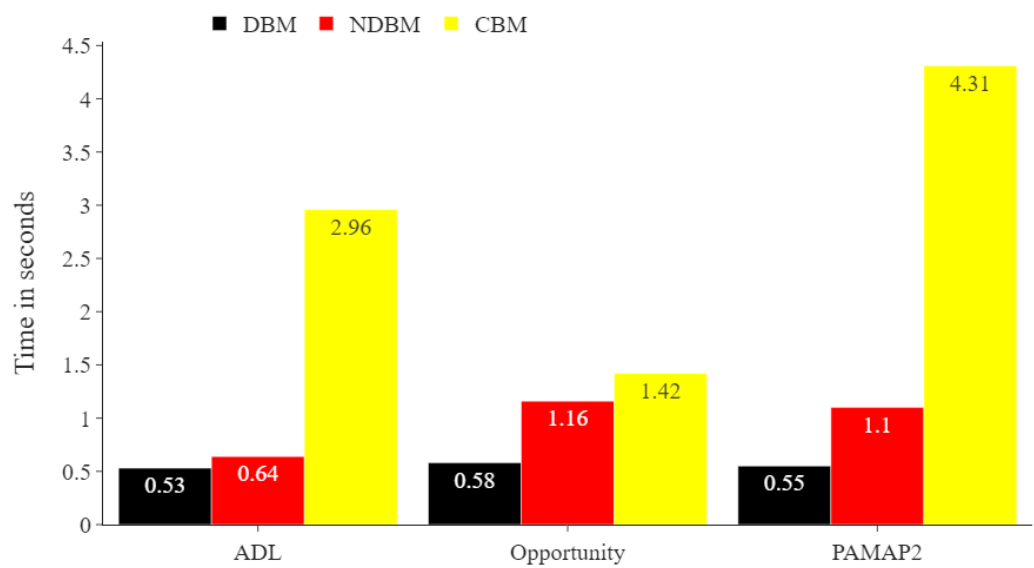

Figure 8. Comparing run times in seconds of the proposed DBM and CBM for all training datasets. The number of samples in the training sets for the ADL, Opportunity, and PAMAP2 datasets were $11,776,1569$, and 6450 , respectively.

\subsection{Statistical Analysis}

A statistical analysis was performed to find out whether there are significant $F 1$ performance differences between the nine sampling methods across five classifiers. The sampling methods analysed are SMOTE, Random_SMOTE, MSMOTE, SMOTE_TomeKLinks, CBSO, ProWSync, as well as the hybrid methods DBM, NDBM, and CBM. The classifiers are LR, RF, SVM, KNN, and MLP. The normality assumption is first estimated using the AndersonDarling normality test on each sampling method and classifier combination [50,52]. This determines whether parametric statistical analysis, such as ANOVA, may be used in the 
case of normality, or a non-parametric method, such as the Friedman test, in the case of non-normality [53].

Table 8 shows the results of the Anderson-Darling normality test on sampling methods based on the five classifiers results for each dataset [52]. The mean F1 scores shown are obtained from 45 'samples', where one sample represents one sample method and classifier combination. On the PAMAP2 dataset, the Anderson-Darling $p$-value is more than 0.05 $(\alpha=0.05)$-suggesting the null hypothesis of a normal distribution-and so an ANOVA could then be used. For the ADL- and Opportunity-based results, Table 8 shows a rejection of the null hypothesis $(p<0.05)$ [52]. This indicates that these dataset results are not normally distributed and, therefore, ANOVA cannot be applied [54]. In its place, we use a Friedman test [55].

Table 8. Anderson-Darling normality test on sampling methods based on the 5 classifiers results $\times 9$ sampling methods $(5 \times 9=45$ sample size $)$ on each dataset. The $p$-value is less than $0.05(\alpha=0.05)$ for ADL and Opportunity which suggests that ADL and Opportunity are not normally distributed compared to PAMPA2.

\begin{tabular}{ccccc}
\hline Data & Mean & Standard Deviation & Sample Size & $p$-Value \\
\hline ADL & 0.8840 & 0.0399 & 45 & 0.0007 \\
Opportunity & 0.3773 & 0.0548 & 45 & 0.0000 \\
PAMAP2 & 0.7272 & 0.0406 & 45 & 0.0680 \\
\hline
\end{tabular}

\subsubsection{ANOVA on PAMAP2}

Table 9 reveals that the ANOVA test detected no statistical evidence to reject the null hypothesis $(p>0.05)$. In other words, when using PAMAP2, all sampling methods performed the same and none was found to perform significantly differently to the others.

\subsubsection{Friedman Test on ADL and Opportunity}

The Friedman test in Table 10 indicates that the $p$-values of the data are less than $0.05(\alpha=0.05)$ for the ADL and Opportunity datasets. Therefore, the null hypothesis is then violated. This means that there is a statistically significant difference across the sampling methods. In other words, one or more of the sampling methods can show different influences on these datasets.

Table 9. ANOVA for PAMAP2 dataset.

\begin{tabular}{cccccc}
\hline Data & $\begin{array}{c}\text { Degrees of } \\
\text { Freedom }\end{array}$ & Sum of Squares & Mean Square & F Value & $p$-Value \\
\hline PAMAP2 & 8 & 0.0067 & 0.0008 & 0.4602 & 0.8757 \\
\hline
\end{tabular}

Table 10. Friedman test results indicate that the $p$-value is less than $0.05(\alpha=0.05)$ for the ADL and Opportunity datasets. This means that one or more of the sampling methods is more effective than the others.

\begin{tabular}{cccc}
\hline Data & Degrees of Freedom & Chi-Square & $p$-Value \\
\hline ADL & 8 & 21.8133 & 0.0053 \\
Opportunity & 8 & 24.2133 & 0.0021 \\
\hline
\end{tabular}

Tables 11 and 12 display the ranks drawn from the Friedman test in the ADL and Opportunity datasets [55]. The test compares rankings across the five classifiers (rows) and nine sampling methods (columns). Ranking is conducted for each classifier row, with sampling methods ranked between 1 (lowest) and 9 (high). The tables then summarize the total ranks obtained for each column to obtain the overall ranking for each sampling method [55].

Both Tables 11 and 12 show that CBM has a consistently high ranking compared to the other sampling approaches across a range of classifiers. This supports our earlier finding that $\mathrm{CBM}$ is the highest performer. 
Table 11. Friedman sum-of-ranks test on ADL-based results for all methods and classifiers. CBM is the overall highest ranking method.

\begin{tabular}{|c|c|c|c|c|c|c|c|c|c|}
\hline Classifier & CBSO & NDBM & CBM & DBM & MSMOTE & Pro-WSyn & Random_SMOTE & SMOTE_TomekLinks & SMOTE \\
\hline KNN & 1 & 7 & 9 & 4 & 5 & 8 & 2 & 6 & 3 \\
\hline LR & 1 & 8 & 3 & 9 & 2 & 5 & 6 & 7 & 4 \\
\hline MLP & 3 & 8 & 9 & 7 & 1 & 5 & 2 & 4 & 6 \\
\hline RF & 1 & 6 & 9 & 4 & 7 & 8 & 3 & 5 & 2 \\
\hline SVM & 1 & 8 & 7 & 9 & 2 & 3 & 6 & 4 & 5 \\
\hline Sum of ranks & 7 & 37 & 37 & 33 & 17 & 29 & 19 & 26 & 20 \\
\hline
\end{tabular}

Table 12. Friedman sum-of-ranks test on Opportunity-based results for all methods and classifiers. CBM is the overall highest ranking method.

\begin{tabular}{|c|c|c|c|c|c|c|c|c|c|}
\hline Classifier & CBSO & NDBM & CBM & DBM & MSMOTE & Pro-WSyn & Random_SMOTE & SMOTE_TomekLinks & SMOTE \\
\hline KNN & 5 & 6 & 9 & 7 & 1 & 4 & 8 & 3 & 2 \\
\hline LR & 5 & 9 & 7 & 8 & 1 & 2 & 6 & 4 & 3 \\
\hline MLP & 5 & 7 & 9 & 8 & 1 & 3 & 2 & 4 & 6 \\
\hline RF & 4 & 5 & 8 & 3 & 1 & 9 & 7 & 6 & 2 \\
\hline SVM & 2 & 7 & 8 & 9 & 1 & 4 & 3 & 5 & 6 \\
\hline Sum of ranks & 21 & 34 & 41 & 35 & 5 & 22 & 26 & 22 & 19 \\
\hline
\end{tabular}

\section{Discussion and Future Work}

Prior studies such as [2,4] have highlighted the lack of works that address and investigate the impact of the class imbalance problem in human activity recognition. Our present study fills this gap by proposing three approaches, DBM, NDBM, and CBM, to reduce the class imbalance and substantially improve human activity recognition (HAR) performance. We found that the proposed hybrid sampling methods worked better than applying any single sampling algorithm across three different HAR datasets. The benefit of the proposed approaches is that they generate more diverse samples, and thus improve the generalisability of the learning algorithm.

The cluster-based method (CBM) reveals consistently better performance than distance $(\mathrm{DBM})$ or noise detection (NDBM)-based methods. A Friedman test additionally showed the statistical superiority of CBM over the other methods for two datasets, ADL and Opportunity, across five different classifiers.

Minority classes in particular benefit from using CBM, as shown in Figures 5-7. This indicates that CBM would be a good choice when working with imbalanced HAR data involving activities similar to those found in Opportunity and ADL.

DBM, which is a combination of distance-based SMOTE and Random_SMOTE, provides the next highest performing combination. The main advantage of DBM, however, is that it uses significantly less computational resources than CBM. We suggest this method in instances where the training data suffer from small sample size and there is limited computational power. The main issue with DBM compared to NDBM and CBM is more likely to introduce noisy samples. The DBM does not perform any data filtering such as clustering processes prior to oversampling the data. One way to improve the DBM is to use a technique to assess the similarities between the synthetic samples and the training data samples (i.e., the original data), then to use only the most similar synthetic samples to the original sample in order to oversample the training data. For example, one can use the SMOTE and Random_SMOTE approaches to generate synthetic samples from the original training samples and use an efficient similarity metric such as Euclidean distance to compare the generated synthetic data to the original training samples and then use only the most similar synthetic samples and disregard the least similar. Our reason for this is that it might ensure that high-quality synthetic samples are used to oversample the training data.

Moreover, the key problem with the NDBM is that is relies on sampling methods that eliminate some samples during the oversampling process. This can lead to losing some valuable information of the activities. Therefore, we suggest that CBM be used by 
researchers to ensure they can be able to oversample the imbalance human activity data without losing any information.

To minimise complexity, we opted to use the default settings for most of the classifiers and sampling algorithms. Despite this, we believe that the general findings of the work regarding the influence of sampling on HAR still hold, and we have left further optimisation for future work.

One limitation of our proposed solutions is the choice of features. We chose to adopt time-domain features because these are efficient and fast to compute. This work might be extended by expanding on the feature set to incorporate, say, frequency domain features [12]. Additionally, we only considered data from a single accelerometer and a single location per dataset. How our sampling strategies might perform using an expanded feature set and a larger number of sensors will be the focus of future work.

A further area of future work will be to investigate more complex ensembles of sampling methods, e.g., combing distance with cluster-based methods. One challenge here will be to reduce the computational complexity of the clustering method, while preserving its ability to produce diverse samples.

Author Contributions: F.A. conceived the idea and research methodology as well as developing the proposed approaches, performing the experiments, and writing. J.A.W. contributed to the research by supervising and directing the research and analysis, then reviewing and editing the paper. L.O. contributed by reviewing and providing feedback. All authors have read and agreed to the published version of the manuscript.

Funding: Jamie A. Ward is funded by a Leverhulme supported APEX award from The British Academy, Royal Academy of Engineering and Royal Society (R156910).

Acknowledgments: Fayez Alharbi was supported by Majmaah University through Saudi Arabia Cultural Bureau in United Kingdom.

Conflicts of Interest: The authors declare no conflict of interest.

\section{Appendix A}

Table A1. Parammeters settting of SVM, LR and KNN on multiple dataset. Ref. [47] provides description about the parameters that we used.

\begin{tabular}{ccccc}
\hline Algorithms & Parameters & ADL & Opportunity & PAMAP2 \\
\hline SVM & gamma & 0.1 & 0.1 & 0.1 \\
& C & 20 & 20 & 20 \\
& kernel & rbf & rbf & rbf \\
& max_iter & -1 & -1 & -1 \\
LR & decision_function_shape & ovr & ovr & ovr \\
& multi_class & multinomial & multinomial & multinomial \\
& solver & newton-cg & sag & sag \\
& max_iter & 250 & 250 & 250 \\
KNN & C & 2 & 2 & 2 \\
& penalty & L2 & L2 & L2 \\
& n_neighbors & 3 & 5 & 3 \\
& algorithm & auto & auto & auto \\
\hline
\end{tabular}




\section{Appendix B}

Table A2. Comparing the performance of the baseline classifiers on multiple datasets. The reported mean of $F 1$ scores and ( \pm standard deviation) were obtained from 30 repetitions. The $F 1$ score is as \%.

\begin{tabular}{ccc}
\hline Data & Classifier & F1 Score \\
\hline ADL & KNN & $85.63( \pm 0.043)$ \\
& LR & $84.51( \pm 0.026)$ \\
& MLP & $87.2( \pm 0.047)$ \\
& RF & $82.76( \pm 0.037)$ \\
Opportunity & SVM & $90.76( \pm 0.037)$ \\
& KNN & $31.36( \pm 0.052)$ \\
& LR & $26.03( \pm 0.012)$ \\
& MLP & $28.85( \pm 0.017)$ \\
& RF & $33.15( \pm 0.032)$ \\
& SVM & $34.04( \pm 0.012)$ \\
& KNN & $69.44( \pm 0.033)$ \\
& LR & $64.81( \pm 0.094)$ \\
& MLP & $71.85( \pm 0.081)$ \\
& RF & $71.72( \pm 0.057)$ \\
\end{tabular}

\section{Appendix C}

We exhibited the $F 1$ score of the baseline classifiers including the SVM, RF, LR and $\mathrm{KNN}$ in order to compare the influence of the sampling methods in improving their $F 1$ score. The sampling methods were the proposed DBM, NDBM and CBM as well as the existing methods including, SMOTE, Random_SMOTE, SMOTE_Tomeklinks, MSMOTE, CBSO, and ProWSyn.

The below figures compared the F1 scores of the SVM, RF, LR, and KNN on the Opportunity, PAMAP2, and ADL datasets. For more details about the dataset, see Section 4.

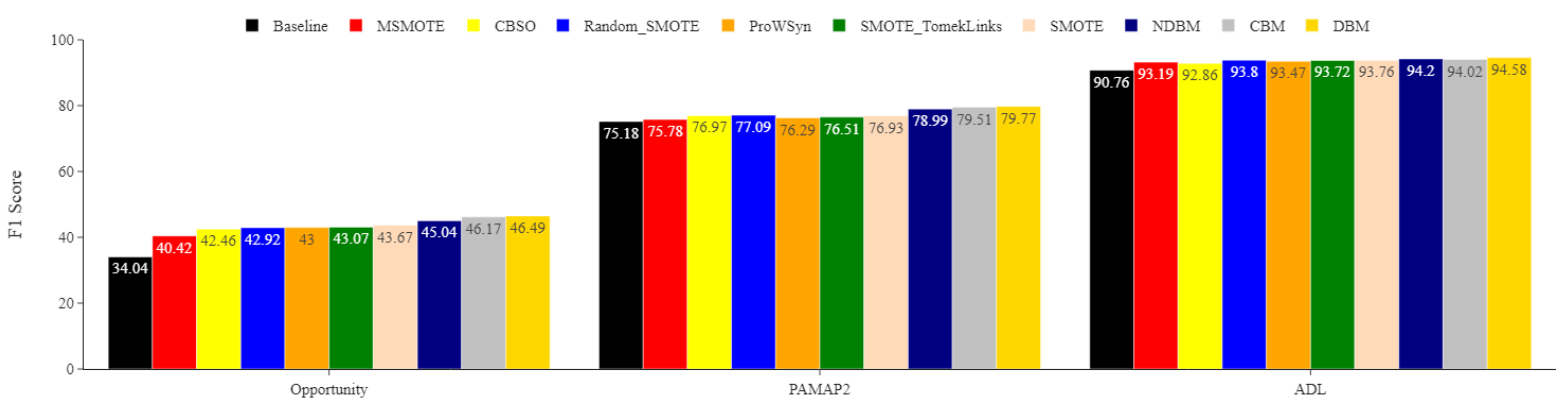

Figure A1. F1 score of baseline (SVM), the proposed method, and the sampling methods for the Opportunity, PAMAP2, and ADL datasets. The reported means of $F 1$ scores were obtained from 30 repetitions.

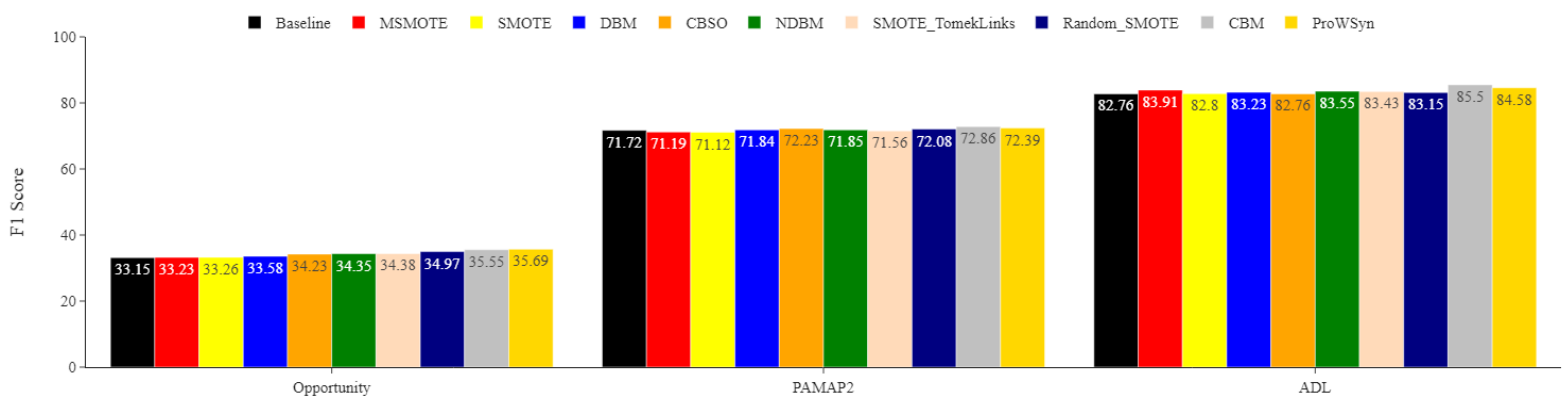

Figure A2. F1 score of baseline (RF), the proposed method, and the sampling methods for the Opportunity, PAMAP2, and ADL datasets. The reported means of $F 1$ scores were obtained from 30 repetitions. 


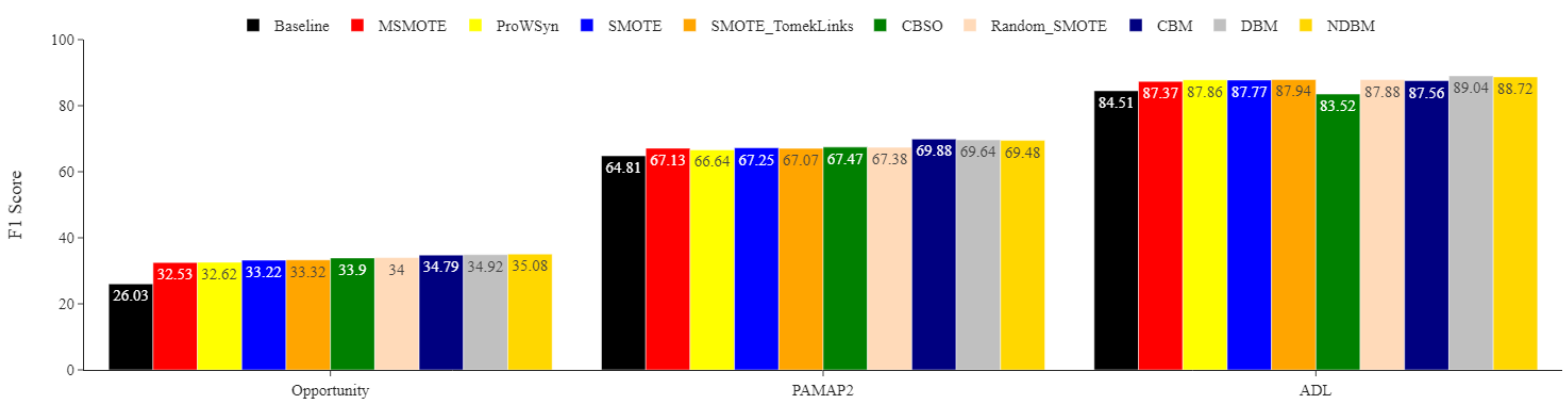

Figure A3. F1 score of baseline (LR), the proposed method, and the sampling methods for the Opportunity, PAMAP2 and ADL datasets. The reported means of $F 1$ scores were obtained from 30 repetitions.

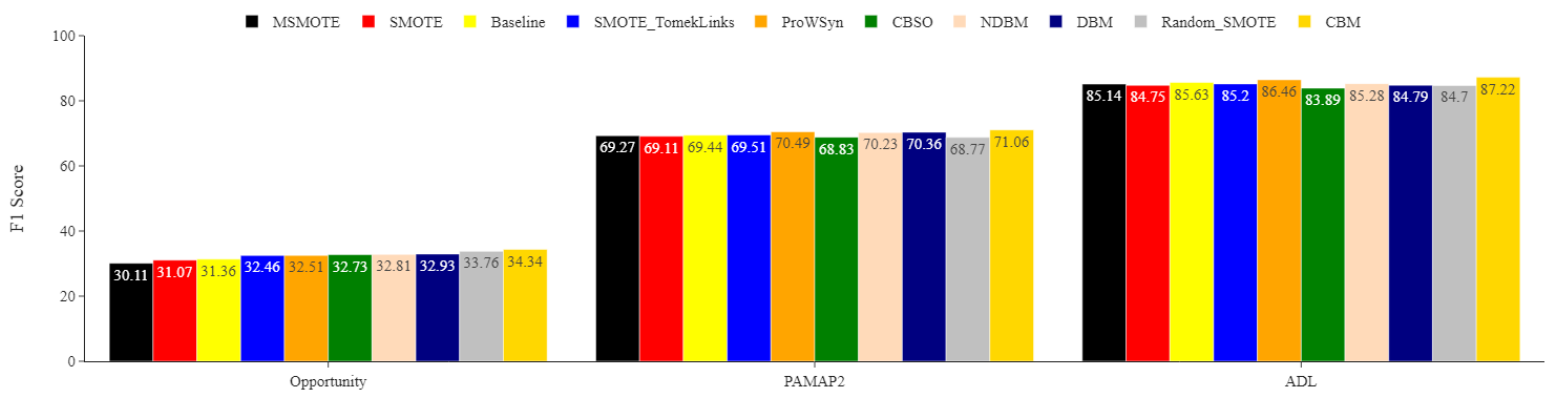

Figure A4. F1 score of baseline (KNN), the proposed method, and the sampling methods for the Opportunity, PAMAP2, and ADL datasets. The reported means of $F 1$ scores were obtained from 30 repetitions.

\section{References}

1. Ordóñez, F.J.; Roggen, D. Deep Convolutional and LSTM Recurrent Neural Networks for Multimodal Wearable Activity Recognition. Sensors 2016, 16, 115. [CrossRef] [PubMed]

2. Chen, K.; Zhang, D.; Yao, L.; Guo, B.; Yu, Z.; Liu, Y. Deep Learning for Sensor-Based Human Activity Recognition: Overview, Challenges, and Opportunities. ACM Comput. Surv. 2021, 54, 1-40. [CrossRef]

3. Bao, L.; Intille, S.S. Activity Recognition from User-Annotated Acceleration Data BT-Pervasive Computing; Springer: Berlin/Heidelberg, Germany, 2004; pp. 1-17.

4. Wang, J.; Chen, Y.; Gu, Y.; Xiao, Y.; Pan, H. SensoryGANs: An Effective Generative Adversarial Framework for Sensor-based Human Activity Recognition. In Proceedings of the 2018 International Joint Conference on Neural Networks (IJCNN), Rio de Janeiro, Brazil, 8-13 July 2018; pp. 1-8.

5. Ward, J.A.; Lukowicz, P.; Troster, G.; Starner, T.E. Activity Recognition of Assembly Tasks Using Body-Worn Microphones and Accelerometers. IEEE Trans. Pattern Anal. 2006, 28, 1553-1567. [CrossRef]

6. Van Laerhoven, K.; Gellersen, H.W. Spine versus porcupine: A study in distributed wearable activity recognition. In Proceedings of the Eighth International Symposium on Wearable Computers, Arlington, VA, USA, 31 October-3 November 2004; Volume 1, pp. 142-149. [CrossRef]

7. Catellier, D.J.; Hannan, P.J.; Murray, D.M.; Addy, C.L.; Conway, T.L.; Yang, S.; Rice, J.C. Imputation of missing data when measuring physical activity by accelerometry. Med. Sci. Sport. Exerc. 2005, 37, S555-S562. [CrossRef] [PubMed]

8. Saeed, A.; Ozcelebi, T.; Lukkien, J. Synthesizing and Reconstructing Missing Sensory Modalities in Behavioral Context Recognition. Sensors 2018, 18, 2967. [CrossRef]

9. Ramamurthy, S.R.; Roy, N. Recent trends in machine learning for human activity recognition-A survey. In Interdisciplinary Reviews: Data Mining and Knowledge Discovery; Wiley Online Library: Hoboken, NJ, USA, 2018; Volume 8, pp. 1-11.

10. Lago, P.; Matsuki, M.; Inoue, S. Achieving Single-Sensor Complex Activity Recognition from Multi-Sensor Training Data. arXiv 2020, arXiv:2002.11284.

11. Anliker, U.; Ward, J.; Lukowicz, P.; Troster, G.; Dolveck, F.; Baer, M.; Keita, F.; Schenker, E.; Catarsi, F.; Coluccini, L.; et al. AMON: A wearable multiparameter medical monitoring and alert system. IEEE Trans. Inf. Technol. Biomed. 2004, 8, 415-427. [CrossRef]

12. Bulling, A.; Blanke, U.; Schiele, B. A tutorial on human activity recognition using body-worn inertial sensors. ACM Comput. Surv. 2014, 46, 1-33. [CrossRef]

13. Kwon, H.; Tong, C.; Haresamudram, H.; Gao, Y.; Abowd, G.D.; Lane, N.D.; Plötz, T. IMUTube: Automatic Extraction of Virtual on-Body Accelerometry from Video for Human Activity Recognition. In Proceedings of the ACM on Interactive, Mobile, Wearable and Ubiquitous Technologies; ACM: New York, NY, USA, 2020; Volume 4. [CrossRef] 
14. Kaur, H.; Pannu, H.S.; Malhi, A.K. A systematic review on imbalanced data challenges in machine learning: Applications and solutions. ACM Comput. Surv. 2019, 52, 4. [CrossRef]

15. Nweke, H.F.; Teh, Y.W.; Mujtaba, G.; Alo, U.R.; Al-garadi, M.A. Multi-sensor fusion based on multiple classifier systems for human activity identification. Human-Centric Comput. Inf. 2019, 9, 1. [CrossRef]

16. He, H.; Garcia, E.A. Learning from imbalanced data. IEEE Trans. Knowl. Data Eng. 2009, 21, 1263-1284.

17. Guo, H.; Li, Y.; Shang, J.; Gu, M.; Huang, Y.; Gong, B. Learning from class-imbalanced data: Review of methods and applications. Expert Syst. Appl. 2017, 73, 220-239.

18. Cook, D.J.; Krishnan, N.C. Activity Learning: Discovering, Recognizing and Predicting Human Behavior from Sensor Data; John Wiley \& Sons: Hoboken, NJ, USA, 2015.

19. Yang, J.; Nguyen, M.N.; San, P.P.; Li, X.L.; Krishnaswamy, S. Deep Convolutional Neural Networks on Multichannel Time Series for Human Activity Recognition. In Proceedings of the Twenty-Fourth International Joint Conference on Artificial Intelligence, Buenos Aires, Argentina, 25-31 July 2015.

20. Chen, Y.; Shen, C. Performance Analysis of Smartphone-Sensor Behavior for Human Activity Recognition. IEEE Access 2017, 5, 3095-3110. [CrossRef]

21. Nweke, H.F.; Teh, Y.W.; Al-garadi, M.A.; Alo, U.R. Deep learning algorithms for human activity recognition using mobile and wearable sensor networks: State of the art and research challenges. Expert Syst. Appl. 2018, 105, 233-261. [CrossRef]

22. Ni, Q.; Fan, Z.; Zhang, L.; Nugent, C.D.; Clel, I.; Zhang, Y.; Zhou, N. Leveraging wearable sensors for human daily activity recognition with stacked denoising autoencoders. Sensors 2020, 20, 5114. [CrossRef]

23. Vandewiele, G.; Dehaene, I.; Kovács, G.; Sterckx, L.; Janssens, O.; Ongenae, F.; De Backere, F.; De Turck, F.; Roelens, K.; Decruyenaere, J.; et al. Overly optimistic prediction results on imbalanced data: A case study of flaws and benefits when applying over-sampling. Artif. Intell. Med. 2021, 111, 101987. [CrossRef]

24. Sousa Lima, W.; Souto, E.; El-Khatib, K.; Jalali, R.; Gama, J. Human Activity Recognition Using Inertial Sensors in a Smartphone: An Overview. Sensors 2019, 19, 3213. [CrossRef]

25. Akbari, A.; Jafari, R. Transferring activity recognition models for new wearable sensors with deep generative domain adaptation In Proceedings of the IPSN 2019-18th International Conference on Information Processing in Sensor Networks, Montreal, QC, Canada, 16-18 April 2019; pp. 85-96.

26. Dietterich, T.G. Ensemble methods in machine learning. In Proceedings of the International Workshop on Multiple Classifier Systems, Cagliari, Italy, 21-23 June 2000; pp. 1-15.

27. Chawla, N.V.; Bowyer, K.W.; Hall, L.O.; Kegelmeyer, W.P. SMOTE: Synthetic minority over-sampling technique. J. Artif. Intell. Res. 2002, 16, 321-357. [CrossRef]

28. Dong, Y.; Wang, X. A new over-sampling approach: Random-SMOTE for learning from imbalanced data sets. Lect. Notes Comput. Sci. 2011, 7091, 343-352.

29. Zhu, X.; Wu, X. Class Noise vs. Attribute Noise: A Quantitative Study. Artif. Intell. Rev. 2004, 22, 177-210. [CrossRef]

30. He, H.; Ma, Y. Imbalanced Learning: Foundations, Algorithms, and Applications, 1st ed.; Wiley-IEEE Press: Hoboken, NJ, USA, 2013.

31. Frenay, B.; Verleysen, M. Classification in the Presence of Label Noise: A Survey. IEEE Trans. Neural Netw. Learn. Syst. 2014, 25, 845-869. [CrossRef] [PubMed]

32. Batista, G.E.A.P.A.; Prati, R.C.; Monard, M.C. A study of the behavior of several methods for balancing machine learning training data. ACM SIGKDD Explor. Newsl. 2004, 6, 20-29. [CrossRef]

33. Hu, S.; Liang, Y.; Ma, L.; He, Y. MSMOTE: Improving classification performance when training data is imbalanced. In Proceedings of the 2nd International Workshop on Computer Science and Engineering: WCSE 2009, Qingdao, China, 28-30 October 2009.

34. Kubat, M.; Matwin, S. Addressing the Curse of Imbalanced Training Sets: One-Sided Selection. In Proceedings of the Fourteenth International Conference on Machine Learning, Nashville, TN, USA, 8-12 July 1997; pp. 179-186.

35. Saez, J.A.; Luengo, J.; Stefanowski, J.; Herrera, F. SMOTE-IPF: Addressing the noisy and borderline examples problem in imbalanced classification by a re-sampling method with filtering. Inf. Sci. 2015, 291, 184-203. [CrossRef]

36. Hoens, T.R.; Chawla, N.V. Imbalanced datasets: From sampling to classifiers. In Foundations, Algorithms, and Applications: In Imbalanced Learning; Wiley: Hoboken, NJ, USA, 2013; pp. 43-59. [CrossRef]

37. Kovács, G. smote-variants: A Python Implementation of 85 Minority Oversampling Techniques. Neurocomputing 2019, 366 , 352-354. [CrossRef]

38. Barua, S.; Islam, M.; Murase, K. ProWSyn: Proximity Weighted Synthetic Oversampling Technique for Imbalanced Data Set Learning. Adv. Knowl. Discov. Data Min. 2013, 7819, 317-328 .

39. Chavarriaga, R.; Sagha, H.; Calatroni, A.; Digumarti, S.T.; Tröster, G.; Millán, J.D.; Roggen, D. The Opportunity challenge: A benchmark database for on-body sensor-based activity recognition. Pattern Recognit. Lett. 2013, 34, 2033-2042. [CrossRef]

40. Reiss, A.; Stricker, D. Creating and benchmarking a new dataset for physical activity monitoring. In Proceedings of the 5th International Conference on PErvasive Technologies Related to Assistive Environments, Crete, Greece, 6-8 June 2012.

41. Casale, P.; Pujol, O.; Radeva, P. Personalization and user verification in wearable systems using biometric walking patterns. Pers. Ubiquitous Comput. 2012, 16, 563-580. [CrossRef]

42. Roggen, D.; Calatroni, A.; Rossi, M.; Holleczek, T.; Förster, K.; Tröster, G.; Lukowicz, P.; Bannach, D.; Pirkl, G.; Ferscha, A.; et al. Collecting complex activity datasets in highly rich networked sensor environments. In Proceedings of the 2010 Seventh International Conference on Networked Sensing Systems (INSS), Kassel, Germany, 15-18 June 2010; pp. 233-240. 
43. Shoaib, M.; Bosch, S.; Incel, O.D.; Scholten, H.; Havinga, P.J.M. Complex human activity recognition using smartphone and wrist-worn motion sensors. Sensors 2016, 16, 426. [CrossRef]

44. Tang, Q.; Vidrine, D.J.; Crowder, E.; Intille, S.S. Automated detection of puffing and smoking with wrist accelerometers. In Proceedings of the PervasiveHealth 2014: 8th International Conference on Pervasive Computing Technologies for Healthcare, Oldenburg, Germany, 20-23 May 2014; pp. 80-87.

45. Lara, O.D.; Labrador, M.A. A survey on human activity recognition using wearable sensors. IEEE Commun. Surv. Tutor. 2013, 15, 1192-1209. [CrossRef]

46. Shoaib, M.; Bosch, S.; Scholten, H.; Havinga, P.J.M.; Incel, O.D. Towards detection of bad habits by fusing smartphone and smartwatch sensors. In Proceedings of the 2015 IEEE International Conference on Pervasive Computing and Communication Workshops, St. Louis, MO, USA, 23-27 March 2015; pp. 591-596.

47. Pedregosa, F.; Varoquaux, G. Scikit-learn: Machine learning in Python. J. Mach. Learn. Res. 2011, 12, $2825-2830$.

48. Sokolova, M.; Lapalme, G. A systematic analysis of performance measures for classification tasks. Inf. Process. Manag. 2009, 45, 427-437. [CrossRef]

49. Pirttikangas, S.; Fujinami, K.; Nakajima, T. Feature Selection and Activity Recognition from Wearable Sensors. In Proceedings of the Third International Conference on Ubiquitous Computing Systems UCS'06, Seoul, Korea, 11-13 October 2006; Springer: Berlin/Heidelberg, Germany, 2006; pp. 516-527. [CrossRef]

50. Ghorbani, R.; Ghousi, R. Comparing Different Resampling Methods in Predicting Students' Performance Using Machine Learning Techniques. IEEE Access 2020, 8, 67899-67911. [CrossRef]

51. Shahi, A.; Deng, J.D.; Woodford, B.J. A streaming ensemble classifier with multi-class imbalance learning for activity recognition. In Proceedings of the 2017 International Joint Conference on Neural Networks (IJCNN), Anchorage, AK, USA, 14-19 May 2017; pp. 3983-3990.

52. Jantschi, L.; Bolboaca, S.D. Computation of Probability Associated with Anderson-Darling Statistic. Mathematics 2018, 6, 88. [CrossRef]

53. Demšar, J. Statistical Comparisons of Classifiers over Multiple Data Sets. J. Mach. Learn. Res. 2006, 7, 1-30.

54. Fisher, R.A. Statistical Methods and Scientific Inference; Hafner Publishing Co.: Oxford, UK, 1956; p. viii 175.

55. Kaur, A.; Kaur, I. An empirical evaluation of classification algorithms for fault prediction in open source projects. J. King Saud-Univ.-Comput. Inf. Sci. 2018, 30, 2-17. [CrossRef] 